Check for updates

Cite this: J. Mater. Chem. A, 2021, 9 , 22462

Received 14th June 2021

Accepted 20th September 2021

DOI: $10.1039 / \mathrm{d} 1 \mathrm{ta0} 05015 \mathrm{j}$

rsc.li/materials-a

\title{
Early-stage decomposition of solid polymer electrolytes in Li-metal batteries $\uparrow$
}

\author{
Edvin K. W. Andersson, (D) a Christofer Sångeland, (D) a Elin Berggren, (D) ${ }^{\mathrm{b}}$ \\ Fredrik O. L. Johansson, (D) cd Danilo Kühn, ${ }^{\mathrm{C}}$ Andreas Lindblad, (iD b \\ Jonas Mindemark (iD a and Maria Hahlin (iD *ab
}

\begin{abstract}
Development of functional and stable solid polymer electrolytes (SPEs) for battery applications is an important step towards both safer batteries and for the realization of lithium-based or anode-less batteries. The interface between the lithium and the solid polymer electrolyte is one of the bottlenecks, where severe degradation is expected. Here, the stability of three different SPEs - poly(ethylene oxide) (PEO), poly( $\varepsilon$-caprolactone) (PCL) and poly(trimethylene carbonate) (PTMC) - together with lithium bis(trifluoromethanesulfonyl)imide (LiTFSI) salt, is investigated after they have been exposed to lithium metal under UHV conditions. Degradation compounds, e.g. $\mathrm{Li}-\mathrm{O}-\mathrm{R}$, $\mathrm{LiF}$ and $\mathrm{Li}_{x} \mathrm{~S}_{y} \mathrm{O}_{z}$, are identified for all SPEs using soft $\mathrm{X}$-ray photoelectron spectroscopy. A competing degradation between polymer and salt is identified in the outermost surface region $(<7 \mathrm{~nm})$, and is dependent on the polymer host. PTMC:LiTFSI shows the most severe decomposition of both polymer and salt followed by PCL:LiTFSI and PEO:LiTFSI. In addition, the movement of lithium species through the decomposed interface shows large variation depending on the polymer electrolyte system.
\end{abstract}

\section{Introduction}

Concerns about the safety and stability of liquid electrolytes based on flammable organic solvents in Li-ion batteries (LIBs) have prompted a search for alternatives. ${ }^{\mathbf{1 , 2}}$ This has led to a recent surge in interest in solid polymer electrolytes (SPEs), where a polymer host material constitutes a high-molecularweight solvent for the lithium electrolyte salt. ${ }^{3-5}$ Furthermore, replacing the liquid electrolyte with a solid membrane, which acts as a barrier for dendrite growth, permits the implementation of lithium metal anodes. ${ }^{5}$ However, similar to liquid electrolyte solvents, the operating potential of lithium metal lies outside of the electrochemical stability window of SPEs. Consequently, the SPE is not thermodynamically stable during battery operation and degrades at the lithium metal electrode interface to form a degradation layer that has a direct influence on the performance and long-term stability of the battery. ${ }^{6-10} \mathrm{It}$

\footnotetext{
${ }^{a}$ Department of Chemistry, Angström Laboratory, Uppsala University, SE-751 21 Uppsala, Sweden. E-mail: maria.hahlin@kemi.uu.se

${ }^{b}$ Department of Physics and Astronomy, Uppsala University, SE-751 21 Uppsala, Sweden

'Institute for Methods and Instrumentation in Synchrotron Radiation Research PSISRR, Helmholtz-Zentrum Berlin für Materialien und Energie, Albert-Einstein-Strasse 15, 12489 Berlin, Germany

${ }^{d}$ Institut für Physik und Astronomie, Universität Potsdam, Karl-Liebknecht-Strasse 2425, 14476 Potsdam, Germany

$\dagger$ Electronic supplementary information (ESI) available. See DOI: $10.1039 /$ d 1 ta05015j
}

is thus important that this layer becomes passivating while still allowing for efficient Li-ion transport. Careful consideration of the degradation processes and the formed degradation layer is essential for successful SPE development and implementation, and could very well usher in a new paradigm in LIB energy densities.

Extensive investigation, primarily by photoelectron spectroscopy (PES) using both soft and hard X-rays (SOXPES, HAXPES), has been and is continuously performed to identify the degradation in the interphase region for liquid electrolytes. $^{\mathbf{1 1 , 1 2}}$ For polymer electrolytes, due to the experimental challenges involved, there are only a few pioneering studies, including work from both our own group and others. ${ }^{13-15}$ While these ex situ studies have allowed characterization of the chemical nature of the passivation layers on lithium metal after they have been formed, complementary techniques are necessary to gain additional insights into the operating reaction mechanisms. The detection of gaseous species as they are formed during the degradation reactions, ${ }^{\mathbf{1 6}}$ for example, has added important pieces to the puzzle of deciphering reaction pathways and polymer stability under these conditions. Our group has also investigated the degradation of polymer hosts in contact with lithium metal (i.e., at extreme low potentials) by computational means using density functional theory (DFT) and $a b$ initio molecular dynamics (AIMD) methods. ${ }^{17,18}$ These efforts have given additional detailed insights into the reactions between lithium metal and ion-conducting polymers, which constitute the initial degradation reactions in lithium metal 
battery systems. One complicating factor of experimentally replicating these computational studies, however, is the extreme reactivity of lithium metal. This reactivity makes it unlikely that the surface of a piece of lithium metal will ever practically be pure lithium metal and the surface of, e.g., lithium foil will not accurately represent a chemical environment where fresh lithium is generated, such as in an operating lithium metal cell. A key challenge for studying the initial degradation reactions of these systems with PES is thus to create a chemical environment that accurately represents a pure lithium metal surface. These conditions can instead be mimicked through the method introduced by Wenzel et al., where a thin layer of lithium metal is applied on the electrolyte surface in situ, after which the degradation can be followed by PES measurements through the electron-transparent lithium film. ${ }^{19}$ Here, we have applied this approach in order to replicate the conditions in our earlier modeling work by studying the initial degradation reactions between three ion-conducting polymers and lithium metal. Several different polymer and salt degradation products are identified in the interface layer using SOXPES. Going beyond the previous DFT and AIMD findings, we have investigated poly(ethylene oxide) (PEO), poly(e-caprolactone) (PCL) and poly(trimethylene carbonate) (PTMC) both as pure polymers and complexed with lithium bis(trifluoromethanesulfonyl)imide (LiTFSI) salt. Including the salt is of extreme interest since it is an essential component of the electrolyte, and is itself prone to degrade.

\section{Results and discussion}

In terms of stability, one of the crucial interfaces in a Li-metal or an anode-less battery is the interface where Li-ion reduction occurs. This is firstly due to the low potential for lithium plating being outside of the stability window of the SPE which during battery operation allows for direct reduction of the SPE, and secondly because of the reactivity of lithium atoms themselves. Here we target the latter, in particular the reaction between lithium and three different SPEs: polyether-, polyester- and polycarbonate-based SPEs with LiTFSI salt. The structural formulae of the polymers as well as the LiTFSI salt are shown in a)<smiles>CCOCCOC</smiles>

c)<smiles>O=C(O[AlH])OCCC=[Tl]</smiles>

PTMC b)

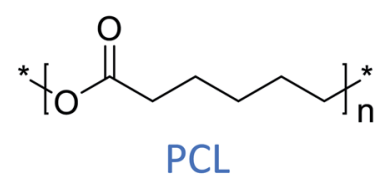

d)

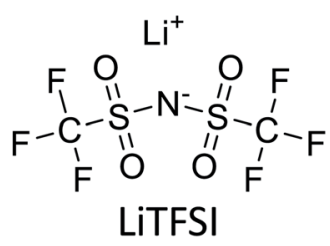

Fig. 1 Molecular structure of (a) poly(ethylene oxide) (PEO), (b) poly( $\varepsilon^{-}$ caprolactone) (PCL), (c) poly(trimethylene carbonate) (PTMC) and (d) lithium bis(trifluoromethanesulfonyl)imide (LiTFSI) salt.
Fig. 1. The use variable energy soft x-rays allowed us to probe the chemical environment of only the uppermost atomic layers. Single bunch operation combined with the ArTOF spectrometer provide high transmission with comparably lower flux than standard undulator beamlines with hemispherical analyzers. This minimizes potential radiation damage to soft-matter samples.

Following in situ deposition of lithium metal under UHV and at ambient temperature conditions, triggering interfacial degradation, PES spectra of the O 1s, C 1s, and Li 1s core levels were measured for each polymer and SPE. In addition, for each SPE also the F 1s, N 1s, and S 2p core levels were measured. PES measurements were performed both before and after Li deposition, and the results are presented in Fig. 2, 3, 5, and 6. The PES spectra are calibrated versus the $\mathrm{CF}_{3}$-peak positioned at a binding energy of $293 \mathrm{eV} \cdot{ }^{\mathbf{1 4}}$ The PES spectra obtained before lithium deposition are deconvoluted based on the molecular structure and using references. The peaks are assigned as follows; in the F 1s spectra a F-C (salt) peak at $\sim 688 \mathrm{eV},{ }^{\mathbf{1 4}}$ in the $\mathrm{O} 1 \mathrm{~s}$ spectra a $\mathbf{O}=\mathrm{C}$ peak at $\sim 532 \mathrm{eV},{ }^{15}$ and a $\mathrm{S}=\mathbf{O}$ (salt) peak at $\sim 532 \mathrm{eV},{ }^{14}$ a C-O peak at $\sim 533 \mathrm{eV},{ }^{15}$ in the $\mathrm{N} 1$ s spectra a $\mathrm{SO}_{2}-\mathbf{N}-$ $\mathrm{SO}_{2}$ (salt) at $\sim 399.5 \mathrm{eV}$, in the $\mathrm{C} 1 \mathrm{~s}$ spectra a hydrocarbon peak $(\mathbf{C}-\mathbf{C} / \mathbf{C}-\mathrm{H})$ at $\sim 285 \mathrm{eV},{ }^{15}$ a $\mathbf{C}-\mathrm{O}$ peak at $\sim 287 \mathrm{eV},{ }^{15}$ a $\mathrm{O}-\mathrm{C}=\mathrm{O}$ peak at $\sim 289 \mathrm{eV},{ }^{20}$ a O- $(\mathbf{C}=\mathrm{O})-\mathrm{O}$ peak at $\sim 290 \mathrm{eV},{ }^{15}$ a $\mathbf{C}-\mathrm{F}_{3}$ (salt) peak at $\sim 293 \mathrm{eV}$, in the $\mathrm{S} 2 \mathrm{p}$ spectra a spin orbit split $\mathrm{N}-\mathrm{S}-\mathrm{O}_{2}$ (salt) peak with the $\mathrm{S} 2 \mathrm{p}_{3 / 2}$ at $169 \mathrm{eV}$, and in the Li 1s spectra a $\mathrm{Li}^{+}$peak at $\sim 56.5 \mathrm{eV}$. All these characteristic peaks for the polymers and the salt have been labelled in Fig. 2-4. The additional peaks that emerge after lithium deposition are identified and discussed further below, and are in the figures indicated by a pink color.

\section{PEO}

Before lithium deposition, both the PEO and the PEO:LiTFSI spectra fit well with that expected from the molecular structure; the PEO spectra are dominated by $\mathrm{C}-\mathbf{O}$ and $\mathbf{C}-\mathrm{O}$ peaks in the $\mathrm{O}$ $1 \mathrm{~s}$ and $\mathrm{C} 1 \mathrm{~s}$ spectra respectively. In addition to this, also adventitious carbon is present, as expected for samples prepared ex situ. For PEO:LiTFSI, these peaks are accompanied by the characteristic LiTFSI peaks $\mathrm{C}-\mathbf{F}, \mathbf{O}=\mathrm{S}=\mathbf{O}, \mathbf{N}-\mathrm{S}, \mathbf{C}-\mathrm{F}_{3}$, and $\mathrm{O}=\mathbf{S}=\mathrm{O}$ peaks. The $\mathbf{O}=\mathrm{S}=\mathbf{O}$ peak is included in the fit of the C-O peak, as they have almost overlapping binding energy. ${ }^{\mathbf{1 4 , 1 5}}$ Large changes are observed in the spectra upon lithium deposition, which show that both the salt and the polymer chains degrade upon contact with lithium.

Starting with the pure $\mathrm{PEO}$, a large decrease in the $\mathbf{C}-\mathrm{O}$ peak intensity relative to the $\mathbf{C}-\mathbf{C} / \mathbf{C}-\mathrm{H}$ peak is observed in the $\mathrm{C} 1 \mathrm{~s}$ spectrum. In addition, a new $\mathrm{O} 1 \mathrm{~s}$ peak emerges at $530.4 \mathrm{eV}$ after lithium deposition, see Fig. 2. This $\mathrm{O} 1 \mathrm{~s}$ peak is, based on its position, assigned to Li-O-R. ${ }^{14}$ This clearly shows that some (but not all) of the $\mathrm{C}-\mathrm{O}$ bonds in PEO are breaking upon contact with metallic lithium, forming $\mathrm{Li}$ alkoxides. In contrast, computational AIMD simulations of PEO oligomers on lithium metal surfaces predict a strong thermodynamic driving force to form $\mathrm{Li}_{2} \mathrm{O}$ along with $\mathrm{C}_{2} \mathrm{H}_{2}$ and $\mathrm{H}_{2}$ gas. ${ }^{18}$ In the PES spectra for PEO no $\mathrm{Li}_{2} \mathrm{O}$ peak is observed. That this stage in the 

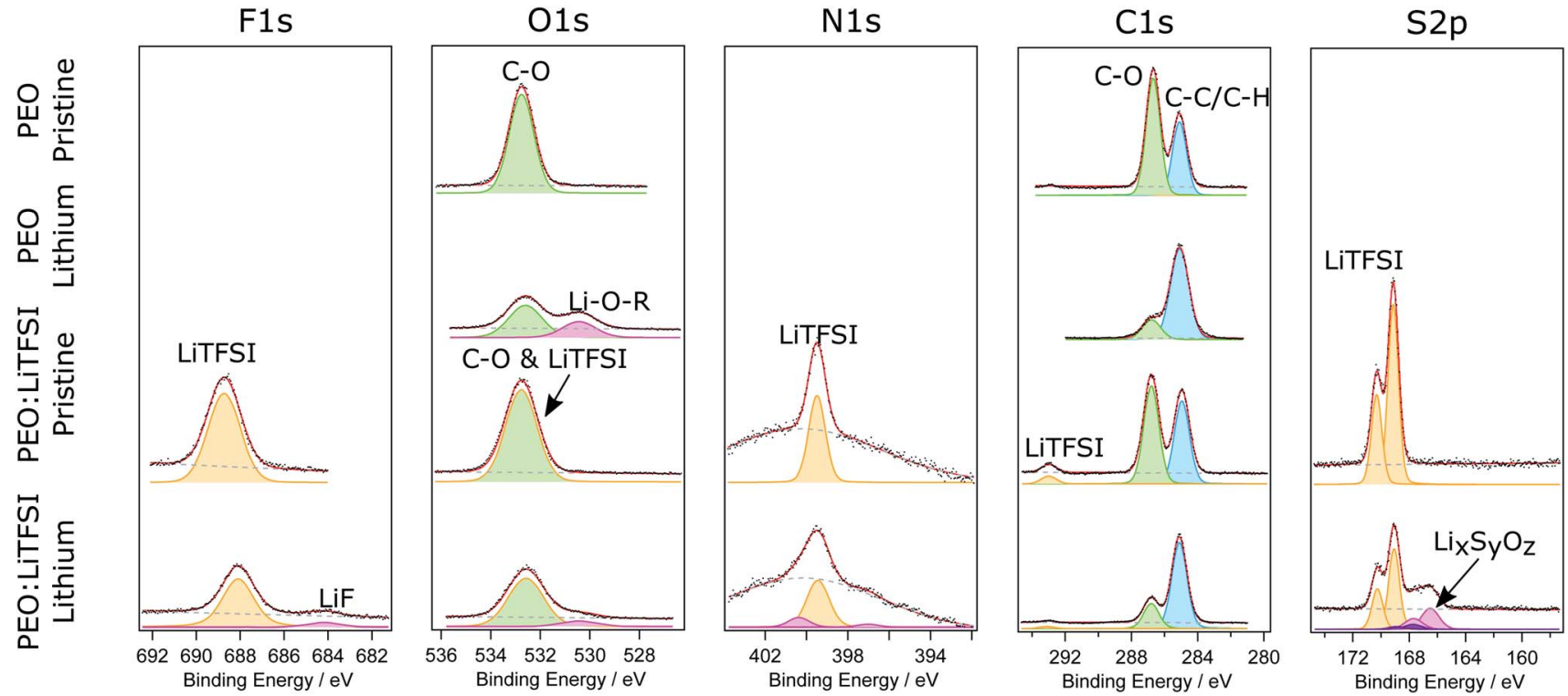

Fig. 2 F 1s, O 1s, N 1s, C 1s, S 2p, and Li 1s spectra for PEO and PEO:LiTFSI before and after lithium deposition. Green: expected of polymer, blue: hydrocarbons, orange: expected of salt, pink: degradation peaks (not present in the pristine sample), purple: unidentified degradation products.

decomposition is not reached to a larger extent indicates a kinetic barrier for the reaction. The formation of Li alkoxides instead of $\mathrm{Li}_{2} \mathrm{O}$ is possibly a consequence of the longer polymer chains - compared to the simulations - "trapping" the decomposition at the Li alkoxide stage. The relative intensity between the $\mathbf{C}-\mathbf{O}$ and the $\mathrm{Li}-\mathbf{O}-\mathrm{R}$ in the $\mathrm{O} 1 \mathrm{~s}$ spectra is $2: 1$ and indicates that in the surface region (a few $\mathrm{nm}$ ) approximately a third of the $\mathrm{O}-\mathrm{C}$ bonds in the PEO have broken, assuming a uniform surface composition.

Moving further to the PEO:LiTFSI film, similar degradation of the PEO is observed also when the salt is present. The intensity ratio between the $\mathbf{C}-\mathrm{O}$ relative to the $\mathbf{C}-\mathbf{C} / \mathbf{C}-\mathrm{H}$ peak decreases to a similar extent as for the pure PEO. However, it is noticed that the relative intensity difference of the $\mathrm{C}-\mathbf{O}$ peak relative to Li-O-R is different for PEO and PEO:LiTFSI. To some degree this is explained by the presence of the salt, were the $\mathrm{S}=$ $\mathbf{O}$ is expected to have a similar binding energy as the C-O peak.

In addition to degradation of the PEO, degradation of the TFSI anion is also observed in the PEO:LiTFSI film. This is seen by the appearance of a LiF peak at $\sim 684.1 \mathrm{eV}$ in the $\mathrm{F} 1 \mathrm{~s}$ spectrum $^{14}$ as well as two new $\mathrm{S} 2 \mathrm{p}$ features at $167.6 \mathrm{eV}$ and $166.6 \mathrm{eV}$, respectively. The assignment of the two new $\mathrm{S} 2 \mathrm{p}$ peaks are not
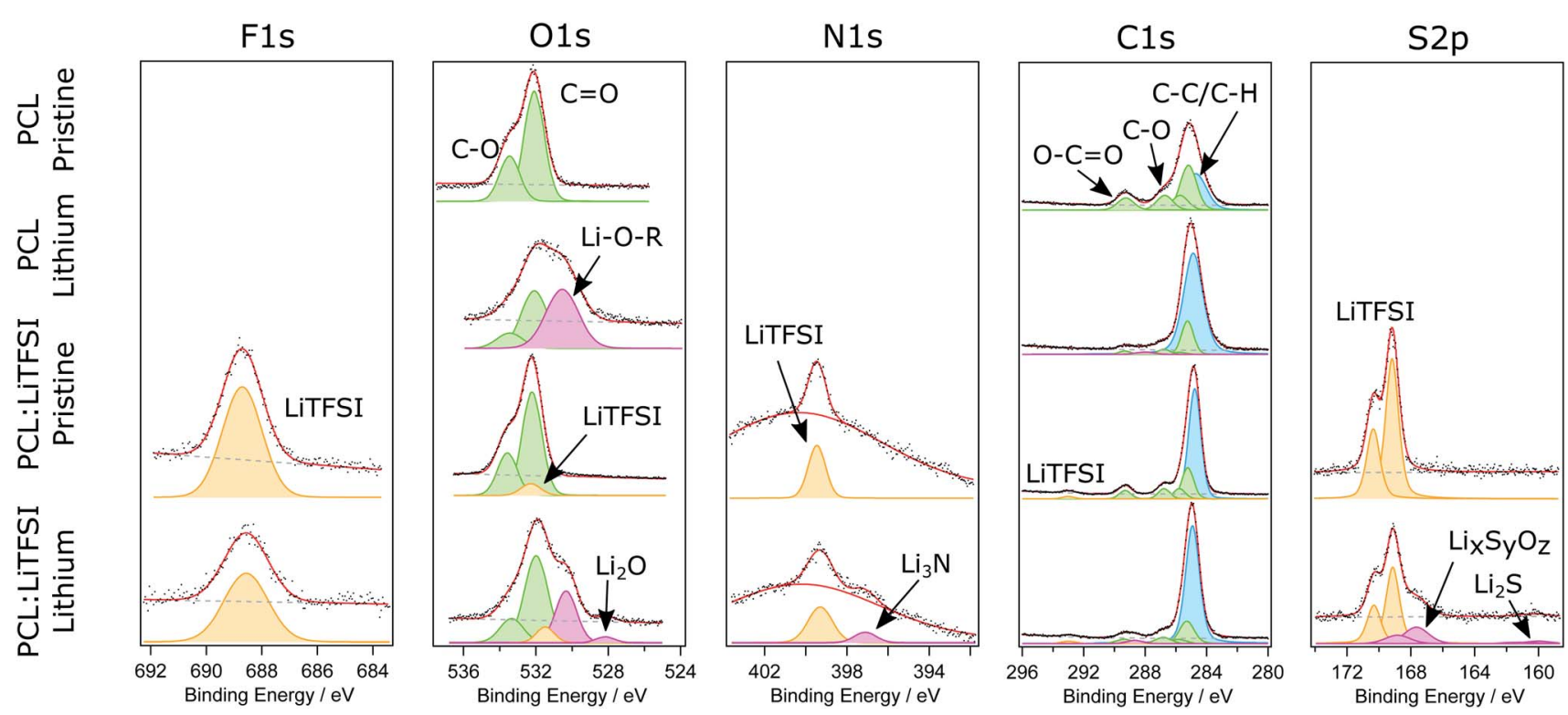

Fig. $3 \mathrm{~F}$ 1s, O 1s, N 1s, C 1s, S 2p, and Li 1s spectra for PCL and PCL:LiTFSI before and after lithium deposition. Green: expected of polymer, blue: hydrocarbons, orange: expected of salt, pink: degradation peaks (not present in the pristine sample). 
a)<smiles>[CH]CCCCCC([O-])OC</smiles>

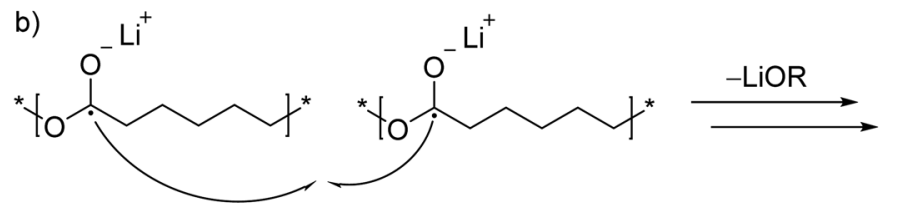

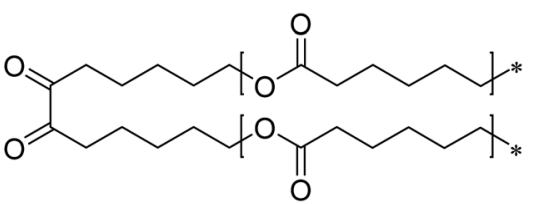

2

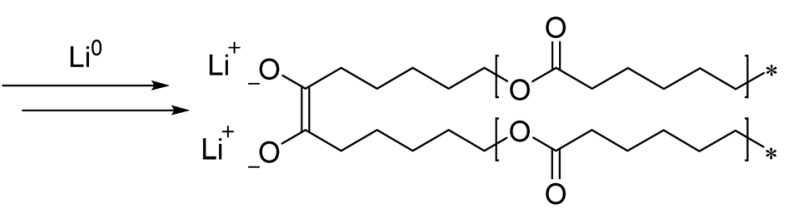

Fig. 4 Illustration of possible reaction pathways by the interaction of PCL with metallic lithium.

clear; however, the position of the peak at $167.6 \mathrm{eV}$ coincides with a feature previously attributed to $\mathrm{Li}_{2} \mathrm{SO}_{3} \cdot{ }^{14}$ However, we note that the formation of the $\mathrm{SO}_{3}$ fragment from the $\mathrm{SO}_{2}$ moieties present in the TFSI anion would require the abstraction of an additional oxygen atom from another source, which we deem unlikely. As there are a range of possible sulfur oxyanions with a range of oxidation numbers for sulfur, we have opted to label this peak as $\mathrm{Li}_{x} \mathrm{~S}_{y} \mathrm{O}_{z}$ to reflect its uncertain origin. After lithium deposition, the $\mathrm{N}$ 1s peak has a broader FWHM that suggest multiple components. This supports the finding of salt degradation. The relative ratio between the $\mathrm{LiF}$ and the $\mathrm{C}-\mathrm{F}$ component, as well between the degradation peaks in $S 2 p$ relative to the TFSI anion are both small. Together with the relatively large decomposition observed for the PEO, this suggests a preferential decomposition of the PEO chains as compared to the TFSI anion for the PEO:LiTFSI system. Previous studies have shown a strong correlation between the formation of TFSI-derived compounds (e.g., $\mathrm{LiF}$ and $\mathrm{Li}_{3} \mathrm{~N}$ ) and the concentration of $\mathrm{H}_{2} \mathrm{O}$ in the electrolyte. ${ }^{14,15,21,22}$ However, the absence of a prominent LiOH peak, suggests negligible quantities of $\mathrm{H}_{2} \mathrm{O}$. This being said, given the hydrophilic quality of PEO, greater TFSI decomposition can be expected under less strict atmospheric conditions. Nevertheless, the degradation species observed in this work closely match those previously reported between PEO:LiTFSI and lithium metal foil. ${ }^{\mathbf{1 4 , 1 5}}$

\section{PCL}

The spectra of PCL and PCL:LiTFSI in Fig. 3 also have a good match with that expected from the structural formulae of the compounds in the sample, both in terms of peak positions and relative intensities. In addition to the peaks seen for $\mathrm{PEO} /$ PEO:LiTFSI, the characteristic $\mathrm{C}$ 1s peak from $\mathrm{O}-\mathrm{C}=\mathrm{O}$, specific to PCL, can also be seen at $289.3 \mathrm{eV} \cdot{ }^{20}$ As expected, large changes are also observed for the PCL-based system upon lithium deposition, and these clearly show degradation of both PCL polymer and salt.

For the pure PCL sample, a large decrease in the $\mathbf{C}-\mathrm{O}$ and $\mathrm{O}-$ $\mathbf{C}=\mathrm{O}$ peak intensities relative to the $\mathbf{C}-\mathbf{C} / \mathbf{C}-\mathrm{H}$ peak is observed in the $\mathrm{C}$ 1s spectra. The $\mathbf{C}-\mathbf{C} / \mathbf{C}-\mathbf{H}$ peak is after lithium deposition completely dominating the spectra, making an accurate deconvolution difficult. At the same time a Li-O-R peak shows up in the $\mathrm{O} 1 \mathrm{~s}$ spectrum, constituting approximately half of the intensity. Alkoxide species may be formed by the breaking of the $\mathrm{C}-\mathrm{O}$ bonds, as previously suggested by computational work. ${ }^{17}$ However, the large quantity of lithium alkoxide observed in the $\mathrm{O}$ 1s spectra following lithium deposition can only explained by the breaking of the $\mathrm{C}-\mathrm{O}$ bond in addition to reduction of the $\mathrm{C}=\mathrm{O}$ bond. Reductions of carboxylic esters to form alkoxides are ubiquitous reactions in organic chemistry, but generally rely on addition of hydrogen in the form of, e.g., hydrides, something which is not available in the studied system. However, as illustrated in Fig. 4a, direct reduction of the carbonyl group of carboxylic esters by reaction with between alkali metals may form the free-radical intermediate $\mathbf{1}$. This reactive species may then go on to further react through a multitude of reactions. Radical coupling (Fig. 4b) leads to a reaction pathway that is analogous to acyloin condensation, wherein a diketone species 2 is formed following the release of alkoxide species. This can then be further reduced by the action of lithium metal to form the dialkoxide 3 , thereby effectively converting $\mathrm{C}=\mathrm{O}$ to $\mathrm{Li}-\mathrm{O}-\mathrm{R}$ species.

Moving further to the PCL:LiTFSI sample, similarities with the degradation of both the pure PCL and PEO:LiTFSI are seen. The intensities of the $\mathbf{C}-\mathrm{O}$ and $\mathbf{C}=\mathrm{O}$ peaks decrease relative to the $\mathbf{C}-\mathbf{C} / \mathbf{C}-\mathbf{H}$ peak after deposition, although not to the same extent as for the PCL sample. This suggests less degradation of 
the PCL chains when the salt is present. Similar to the pure PCL, a Li-O-R peak appears also for PCL:LiTFSI. The relative intensity ratio between the $\mathrm{C}-\mathbf{O}$ and $\mathrm{Li}-\mathbf{O}-\mathrm{R}$ peaks is for PCL slightly larger to that of PEO both when salt is present and absent. Degradation of the TFSI anion is observed for PCL:LiTFSI as well, although in general smaller changes are observed for the PCL:LiTFSI compared to PEO:LiTFSI. Specifically, less LiF and $\mathrm{Li}_{x} \mathrm{~S}_{y} \mathrm{O}_{z}$ can be seen. In addition, two new peaks emerge for the PCL:LiTFSI, one at $397.2 \mathrm{eV}$ in the $\mathrm{N} 1 \mathrm{~s}$ spectra that is assigned to $\mathrm{Li}_{3} \mathrm{~N}$, and one at $\sim 528 \mathrm{eV}$ in the $\mathrm{O} 1 \mathrm{~s}$ spectra assigned to $\mathrm{Li}_{2} \mathrm{O}$.

\section{PTMC}

The PTMC sample, as prepared through the standard solution casting methods, proved too thick and insulating to efficiently dissipate the generated charges. Thus, the spectra for this sample were completely dominated by charging both before and after Li deposition and are therefore omitted. Fortunately, the PTMC:LiTFSI film was sufficiently thin to prevent charging, and the spectra are presented in Fig. 5. Before lithium deposition, the spectra of PTMC:LiTFSI in Fig. 5 have a good match with that expected from the structural formulae of the compounds in the sample, both in terms of peak positions and relative intensities. The characteristic $\mathrm{O}-(\mathbf{C}=\mathrm{O})-\mathrm{O}$ specific to PTMC is found at $290.9 \mathrm{eV} .^{15}$

After lithium deposition, there are drastic changes in the surface composition. Compared to the PEO:LiTFSI and PCL:LiTFSI systems these changes appear to be much more extensive. A recent DFT studies have also suggested that PTMC:LiTFSI is more susceptible towards reduction compared to both PEO:LiTFSI and PCL:LiTFSI, respectively, ${ }^{23}$ the polycarbonate PTMC also has a structure with several possible points of attack for bond cleavage. Considering $\mathrm{C}-\mathrm{O}$ bond cleavage to be the main source of polymer fragmentation, PTMC can be cleaved at either side of the carbonyl group, producing shorter $\mathrm{C}_{3}$ species compared to PCL, which is expected to give $\mathrm{C}_{6}$ species through $\mathrm{C}-\mathrm{O}$ bond cleavage. PTMC may also release $\mathrm{CO}_{2}$ through the $\mathrm{C}-\mathrm{O}$ cleavage route, ${ }^{16}$ which should also be a powerful driving force for fragmentation.
Starting with the $\mathrm{O}$ 1s spectrum, the formation of $\mathrm{Li}-\mathbf{O}-\mathrm{R}$ is seen for PTMC:LiTFSI. In addition to this, a $\mathrm{Li}_{2} \mathbf{O}$ peak also appears at $\sim 528.1 \mathrm{eV}$. Together, the $\mathrm{Li}-\mathrm{O}-\mathrm{R}$ and the $\mathrm{Li}_{2} \mathrm{O}$ degradation products are dominating the $\mathrm{O} 1 \mathrm{~s}$ spectrum, and the relative intensity ratios between these and the intact polymer peaks suggest that almost all of the PTMC:LiTFSI is decomposed throughout the surface region, assuming a uniform sample. Severe changes are also observed in the $\mathrm{C} 1 \mathrm{~s}$ spectrum of PTMC:LiTFSI; the characteristic $\mathrm{O}-(\mathbf{C}=\mathrm{O})-\mathrm{O}$ of PTMC has been smeared out towards lower binding energy, and is most likely composed of two different components. The lower binding energy peak is $\sim 290.0 \mathrm{eV}$, tentatively fluorinated species or carbonate anions. These results therefore suggest that one of the $\mathrm{C}-\mathrm{O}$ bonds in PTMC breaks on contact with lithium. Severe salt decomposition is also seen for PTMC:LiTFSI. In comparison with both PEO:LiTFSI and PCL:LiTFSI, the salt decomposition is much more pronounced and other salt decomposition products are also being formed. Similar to PCL:LiTFSI, the $\mathrm{LiF} \mathrm{Li}_{3} \mathrm{~N}$, and $\mathrm{Li}_{x} \mathrm{~S}_{y} \mathrm{O}_{z}$ peaks are observed after lithium deposition on PTMC:LiTFSI. Also, the formation of a new compounds is seen in S 2p spectra, where a large peak emerges at $160.5 \mathrm{eV}$. This peak is assigned to $\mathrm{Li}_{2} \mathrm{~S}$. The intensity ratio between the decomposition products relative to the intact PTMC:LiTFSI components are 1.72 in the $\mathrm{F} 1 \mathrm{~s}$ spectrum, 0.63 in the $\mathrm{N} 1 \mathrm{~s}$ spectrum, and 16.5 in the $\mathrm{S} 2 \mathrm{p}$ spectrum. These differences suggest that the reactions with lithium lead to breakdown of primarily the sulfur center of the TFSI anion, followed by the fluorinated part. However, this omits any gaseous reactions products. The intensity ratio between the LiTFSI and $\mathrm{O}-(\mathbf{C}=\mathrm{O})-\mathrm{O}$ peaks in the $\mathrm{C}$ 1s spectra only changes from 0.47 to 0.34 , indicating that the salt decomposition is a little bit higher than decomposition of the PTMC polymer in the PTMC:LiTFSI sample. Overall, the same decomposition compounds have been reported at the interface between PTMC:LiTFSI and lithium metal foil for post mortem samples, albeit in much smaller quantities. ${ }^{15}$ This indicate that the native oxide film found on lithium metal foil may indeed act as a passivation layer, protecting the PTMC:LiTFSI membrane from severe decomposition. In contrast, deposition of lithium
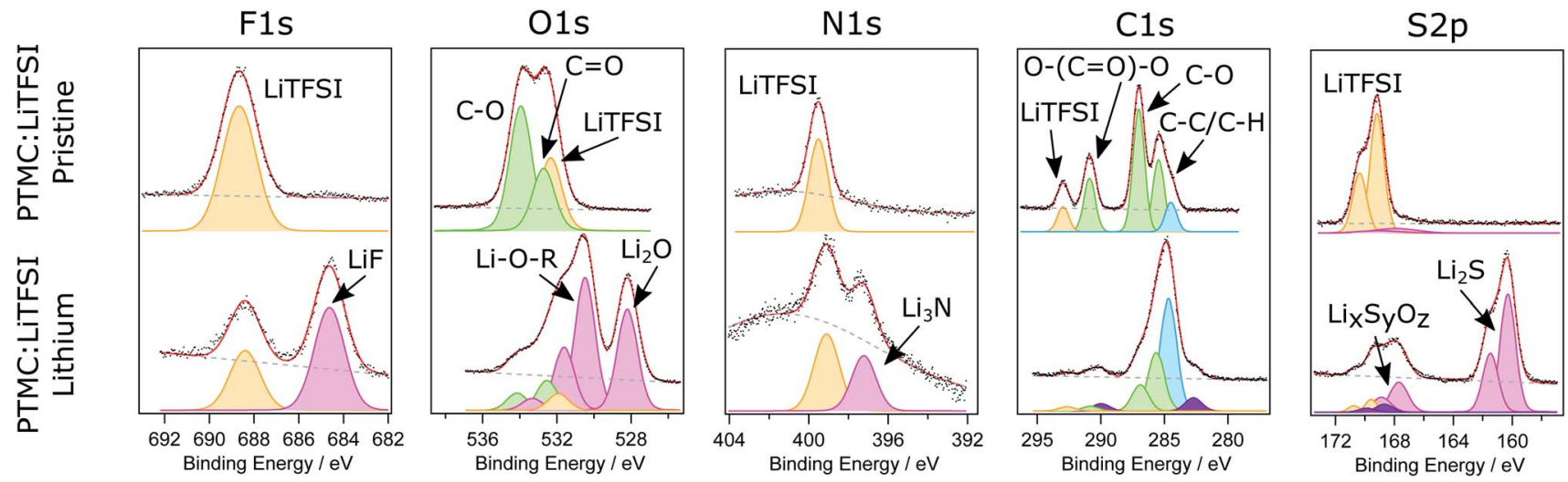

Fig. 5 F 1s, O 1s, N 1s, C 1s, S 2p, and Li 1s spectra for PTMC and PTMC:LiTFSI before and after lithium deposition. Green: expected of polymer, blue: hydrocarbons, orange: expected of salt, pink: degradation peaks (not present in the pristine sample), purple: unidentified degradation products. 
under UVH conditions exacerbates the decomposition of PTMC:LiTFSI, resulting in intense $\mathrm{LiF}, \mathrm{Li}-\mathrm{O}-\mathrm{R}, \mathrm{Li}_{2} \mathrm{O} \mathrm{Li}_{3} \mathrm{~N}$ and $\mathrm{Li}_{2} \mathrm{~S}$ formation.

\section{Evolution of Li 1s peak after lithium deposition}

The Li 1s spectra of the different polymers and SPEs before and at different times after lithium deposition are shown in Fig. 6. A typical broad Li 1s peak is seen for all pristine samples. After lithium deposition, the Li 1s feature shifts approximately $1 \mathrm{eV}$ towards lower binding energy for all samples. With time, the Li 1s feature gradually shifts back towards its original value, and at the same time decrease in intensity. These results indicate that in the deposited metallic lithium is rapidly oxidized and then gradually moves into the bulk of the sample. For PTMC:LiTFSI, contrary to the other SPEs, the lithium metal peak (at $52.9 \mathrm{eV}$ (ref. 24)) is clearly observed directly after lithium deposition. This suggests that the decomposition structure formed in PTMC:LiTFSI is more stable towards lithium or less permeable towards the formed $\mathrm{Li}^{+}$compounds compared to the interface formed on the other SPEs, at least within the time frame of these measurements. It should also be noted that, based on the higher glass transition temperature of PTMC, the chain dynamics are much slower in the PTMC:LiTFSI system than in either PEO:LiTFSI or PCL:LiTFSI, which may be a contributing factor to the observed differences. However, the remarkable stability of the deposited lithium on top of the amorphous PTMC:LiTFSI matrix indicates that some sort of kinetic barrier is additionally in place.

The lithium metal deposition time was equal in all cases; however, the time required for the evolution of the lithium peak is different depending on the SPE. The fastest return towards original intensity and binding energy position is observed for PEO:LiTFSI, where the spectra after $3 \mathrm{~h}$ have almost same intensity as before lithium deposition and with only a small difference in binding energy position. Following is PCL:LiTFSI, where the intensity has decreased approximately half way back to its original value after $\sim 6 \mathrm{~h}$. The peak is however, still to a large extent shifted in binding energy from its original position. The PTMC:LiTFSI sample, on the other hand, still shows a similar main Li $1 \mathrm{~s}$ intensity after $20 \mathrm{~h}$ as directly after lithium deposition and the peak is also shifted in binding energy similar to that of PCL:LiTFSI. To fully understand the diffusion of lithium species more experiments are needed, however these differences clearly show that there are profound differences between the different SPEs.

\section{Carbon depth profile}

A sharp interface between lithium metal and polymer electrolyte is not expected and it is therefore valuable to understand how the degradation spreads to larger depths within the sample. The $\mathrm{C}$ 1s spectrum contains information from both the polymer and the salt, and by following the changes of the $\mathrm{C} 1 \mathrm{~s}$ spectra acquired at different photon energies (i.e. different probing depths), information from different depth are obtained. ${ }^{12}$ The $\mathrm{C}$ 1s PES spectra were recorded with photon energies of: $1000 \mathrm{eV}, 845 \mathrm{eV}, 710 \mathrm{eV}, 600 \mathrm{eV}$ and $370 \mathrm{eV}$, with probing depths of: $6.9 \mathrm{~nm}, 5.7 \mathrm{~nm}, 4.5 \mathrm{~nm}, 3.6 \mathrm{~nm}$ and $1.2 \mathrm{~nm}$, respectively. These values correspond to three times the inelastic mean free path (IMFP) in PCL. The same IMFP is used to approximate the probing depth of PEO and PTMC.

The measurements were performed between 15 to $120 \mathrm{~min}$ after lithium deposition. As a result of this, changes may still occur at the surface region during the time of acquisition. The $\mathrm{C}$ 1s spectra are measured with the smallest and largest probing depth in a rapid succession compared to the time-scale of lithium diffusion, Fig. 6. Intermediate probing depths are measured afterwards. The trends in intensities for different photon energies is used to build a depth profile. It is however important to keep in mind that it is a snapshot of a process rather than a final picture.

In Fig. 7, the $\mathrm{C}$ 1s spectra from the most surface- and bulksensitive measurements are shown for all polymer electrolytes before and after lithium deposition, whilst the intermediate measurements are presented in ESI, Fig. S1. $\dagger$ Fig. 7 also shows the composition for all probing depths, based on the intensities obtained in the deconvolutions. It is clear that with increased probing depth, features in the $\mathrm{C}$ 1s spectra varies in intensity relative to each other. A general trend after lithium deposition is the increase of the hydrocarbon peak intensities relative to the other peaks.
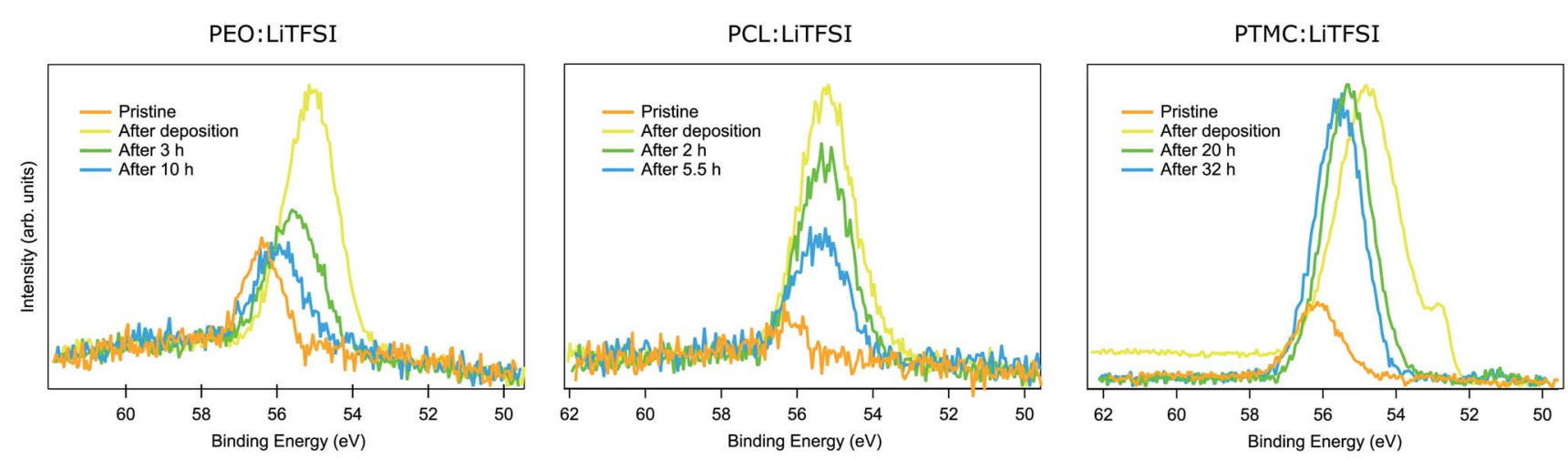

Fig. $6 \mathrm{Li}$ 1s spectra over time for each sample. To facilitate comparison, an intensity shift was applied to the spectra so that the backgrounds overlapped. 
i)
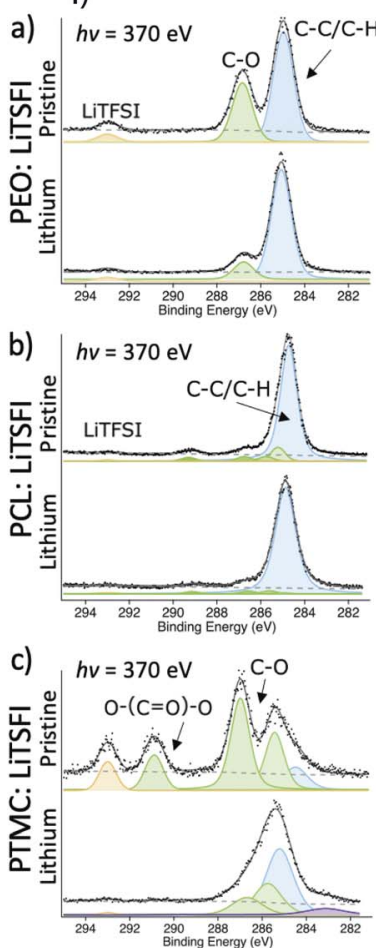
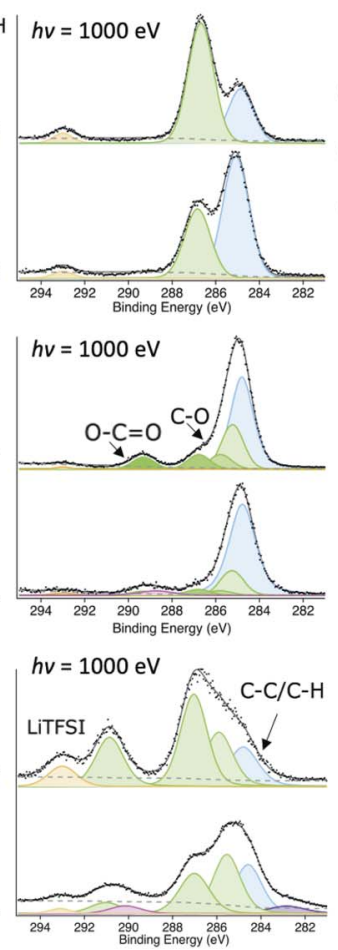

ii)
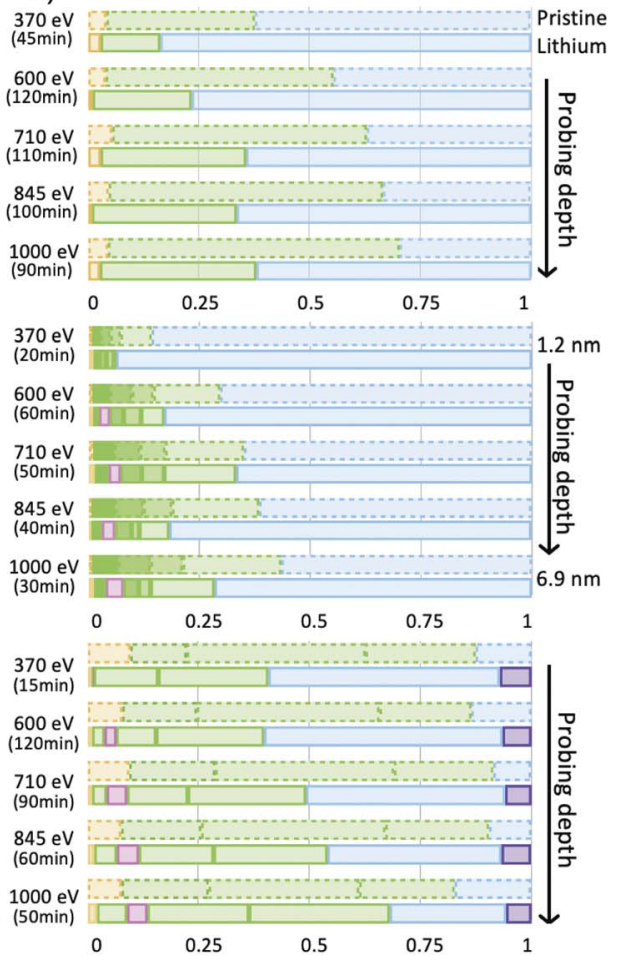

iii)
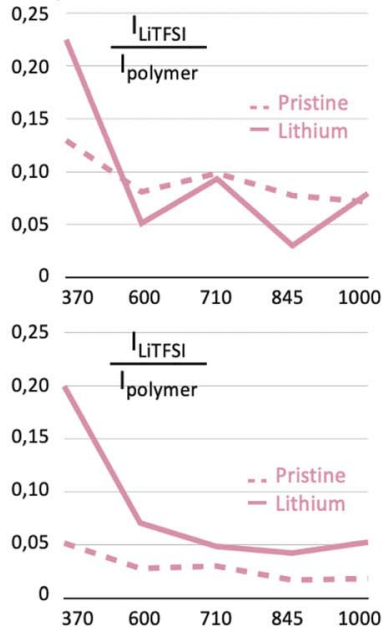

0,25

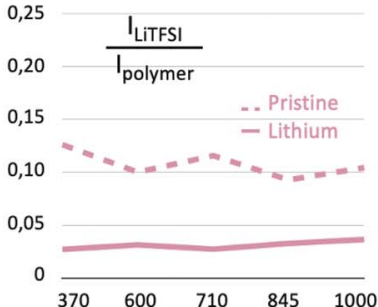

Fig. 7 (i) C 1s core level photoelectron spectra for (a) PEO:LiTFSI, (b) PCL:LiTFSI and (c) PTMC:LiTFSI. Green: polymer, blue: hydrocarbons, orange: LiTFSI, pink: degradation peaks (not present in the pristine sample), purple: unidentified degradation products. (ii) Composition (component intensities from a least squares fit discussed in the text) for each photon energy, before (dashed) and after (solid) Li deposition. (iii) Composition ratio of LiTFSI and polymer where $I_{\text {polymer }}$ is sum of all green peaks for each polymer system.

The C 1s spectra of pristine PEO:LiTFSI, Fig. 7a, shows that the $\mathbf{C}-\mathrm{O}$ peak intensity is increasing relative to the intensity of the $\mathbf{C}-\mathbf{C} / \mathbf{C}-\mathbf{H}$ peak with increased probing depth. This confirms that adventitious carbon is present predominantly at the surface. The relative intensity ratio of the LiTFSI peak compared to the $\mathbf{C}-\mathbf{O}$ is seen to decrease with probing depth, indicating salt accumulation at the surface of the PEO:LiTFSI prior to lithium deposition. Both accumulation of salt and $\mathbf{C}-\mathbf{C} / \mathbf{C}-\mathbf{H}$ has previously been observed for liquid electrolytes, and may thus be expected..$^{25}$ After lithium deposition, the $\mathbf{C}-\mathrm{O}$ peak intensity is still increasing in regard to the $\mathbf{C}-\mathbf{C} / \mathbf{C}-\mathrm{H}$ peak with increasing probing depth. As decomposed PEO forms $\mathbf{C}-\mathbf{C} / \mathbf{C}-\mathbf{H}$ upon contact with lithium, this shows that relatively more intact PEO can be found our found at deeper parts of the sample. As the highest probing depth is $<7 \mathrm{~nm}$, these results show that the decomposition region is, under these experimental conditions, fairly shallow.

Compared to the other samples, PCL:LiTFSI shows a higher concentration of adventitious carbon, both before and after lithium deposition. This, in combination with the overlap of the polymer peaks and the adventitious carbon peak, makes deconvolution of the spectra more difficult. Still, some trends can be observed. For the pristine PCL:LiTFSI sample (Fig. 7b), the $\mathrm{C}$ 1s depth profile shows that the polymer peaks are increasing in intensity relative to the $\mathbf{C}-\mathbf{C} / \mathbf{C}-\mathrm{H}$ peak with increased probing depth, again confirming the adventitious carbon being present mainly on the surface. The intensity ratio between LiTFSI and polymer peaks decreases with depth in this sample as well, indicating salt accumulation at the surface. After lithium deposition the $\mathrm{O}-\mathbf{C}=\mathrm{O}$ and $\mathbf{C}-\mathrm{O}$ peaks have relatively lower intensity compared to the $\mathbf{C}-\mathbf{C} / \mathbf{C}-\mathrm{H}$ peak. With lithium deposited on the sample, the intensities of the polymer peaks are increasing with probing depth, with one exception at a photon energy of $710 \mathrm{eV}$. This irregularity could be due to the dominating concentration of adventitious carbon in combination with overlapping peaks, as discussed in the beginning of this paragraph. Nevertheless, the results show as expected that main decomposition occurs within the same depth region $(<7$ $\mathrm{nm})$ as for PEO:LiTFSI.

The C 1s spectra of pristine PTMC:LiTFSI in Fig. 7c shows that the intensity of the polymer peaks are relatively constant to the $\mathbf{C}-\mathbf{C} / \mathbf{C}-\mathbf{H}$ peak for all probing depth, in contrast to both PEO and PCL. We can however still slightly perceive the decrease of the LiTFSI/polymer intensity ratio, although, compared to PEO and PCL, it is a much smaller difference. The different trends seen for the case of PTMC:LiTFSI may indicate less mobility of both salt and $\mathbf{C}-\mathbf{C} / \mathbf{C}-\mathbf{H}$ species in this polymer. After lithium deposition it is not possible to identify any depth dependence of the salt concentration. However, the trends for the $\mathbf{C}-\mathbf{O}$ and $\mathbf{C}-$ C/C-H peak intensities in the PEO:LiTFSI and PCL:LiTFSI systems after deposition can still be observed in the PTMC:LiTFSI system. The $\mathbf{C}-\mathrm{O}$ peak decreases in intensity relative to the $\mathbf{C}-\mathbf{C} / \mathbf{C}-\mathbf{H}$ after, again indicating less decomposition at higher depths. The unidentified component found at 


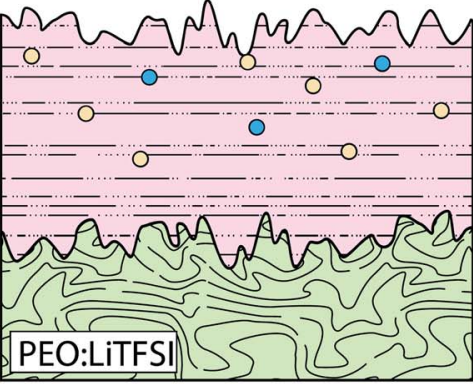

Polymer electrolyte

Decomposed polymer:

ROLi, C-C/C-H

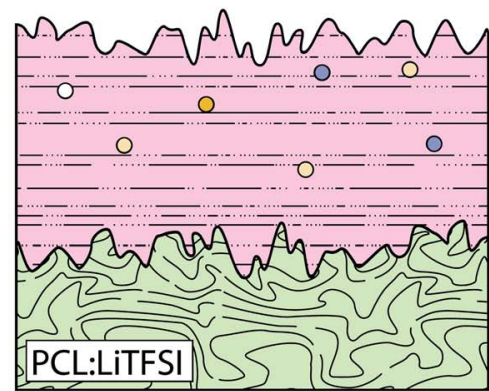

Decomposed salt:

O $\mathrm{Li}_{x} \mathrm{~S}_{y} \mathrm{O}_{z} \quad \mathrm{OLiF}$

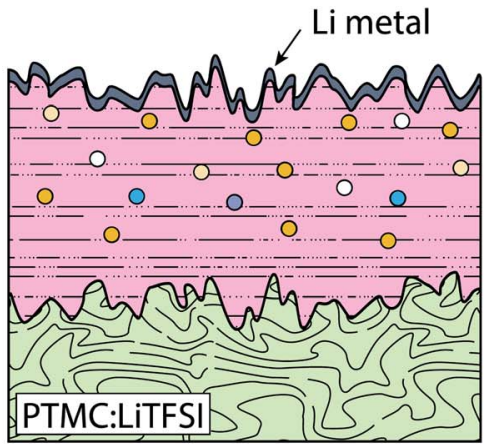

$\mathrm{OLi} 2 \mathrm{~S}$
O LizN

Fig. 8 Illustration of the polymer electrolyte films after lithium deposition. Polymer is green, degradation region is purple, where a darker color indicates a higher degree of polymer degradation, small yellow/blue circles indicate decomposed salt, and lithium is grey.

$283 \mathrm{eV}$ stays constant in intensity over varied probing depth. Like with the other systems, the decomposition region is fairly shallow, within $<7 \mathrm{~nm}$.

A schematic summary of all the PES results is shown in Fig. 8. For all systems both polymers and salt decompose upon contact with lithium. The degree of polymer relative to salt degradation depends on the polymer host. The bare PEO is seen to be more stable than PCL. When the salt is added, PEO still seems more stable than PCL, but at the expense of more severe salt degradation compared to the PCL. Similar degradation compounds are seen in both cases: polymer hydrocarbons, lithium alkoxides $(\mathrm{Li}-\mathrm{O}-\mathrm{C})$ and $\mathrm{Li}_{x} \mathrm{~S}_{y} \mathrm{O}_{z}$. In addition, small quantities of $\mathrm{LiF}$ is seen in the PEO:LiTFSI sample, and $\mathrm{Li}_{2} \mathrm{O}$ and $\mathrm{Li}_{3} \mathrm{~N}$ is seen in small quantities for the PCL:LiTFSI sample. Standing out is the PTMC system, showing much larger quantities of decomposition products from both polymer and salt. Only the PTMC:LiTFSI shows the $\mathrm{Li}_{2} \mathrm{~S}$ decomposition product. $\mathrm{Li}_{2} \mathrm{O}$ could not be detected for PEO or PEO:LiTFSI. AIMD studies have shown that there is a large thermodynamic driving force for $\mathrm{Li}_{2} \mathrm{O}$ formation also for $\mathrm{PEO}$ on contact with lithium metal, ${ }^{18}$ but it appears that the reaction does not proceed all the way to the oxide under these conditions, possibly because of some kinetic limitation. Overall, the degree of decomposition observed for the three different SPE systems agrees well with DFT-based computational work: PTMC is expected to have a lower cathodic stability compared to its polyether- and polyester-based counterparts. ${ }^{23}$ We also note that, in contrast with the semi-crystalline PEO:LiTFSI and PCL:LiTFSI, PTMC:LiTFSI is fully amorphous; since degradation of crystalline structures would require an additional energy input to disrupt these structures, there should be an additional barrier towards degradation of the crystalline domains of these materials. From the analysis of the Li 1s spectra it appears as if PTMC:LiTFSI forms a kinetically more stable interface towards the lithium metal compared to the other SPEs. This puts a finger on the question of how much degradation is too much, and how much is a necessity in order to form a stable, functional interphase. As a final note, it is known that degradation reactions become more severe at elevated temperatures, which is unfortunate given that several of these SPEs require higher temperatures to operate. ${ }^{4}$ One may therefore expect more severe degradation to occur in batteries operating under more realistic conditions. Common for PEO:LiTFSI, PCL:LiTFSI, and possibly also for PTMC:LiTFSI before deposition is that the concentration of the LiTFSI salt is higher at the outermost layers than deeper into the sample. Another trend seen in all spectra is the relative decrease of the $\mathbf{C}-\mathrm{O}$ peak intensity in relation to $\mathbf{C}-\mathbf{C} / \mathbf{C}-$ $\mathrm{H}$ after deposition of lithium. This difference is the most notable at the lowest probing depth $(h v=370 \mathrm{eV})$ and subsides with higher probing depths. The concentration of the decomposition products decreasing with probing depth suggests a decomposition layer thickness of similar or smaller depths than the largest probing depth of $<7 \mathrm{~nm}$.

\section{Conclusions}

The interface between lithium and 3 different SPEs - PEO:LITFSI, PCL:LiTFSI and PTMC:LiTFSI, - was investigated using soft X-ray photoelectron spectroscopy. Both polymer and salt degradation compounds, e.g. $\mathrm{Li}-\mathrm{O}-\mathrm{R}$ and $\mathrm{Li}_{x} \mathrm{~S}_{y} \mathrm{O}_{z}$, were identified at the lithium metal/SPE interface for all SPEs. For PEO:LiTFSI and PTMC:LiTFSI, LiF was detected. For PCL:LiTFSI and PTMC:LiTFSI $\mathrm{Li}_{2} \mathrm{O}$ and $\mathrm{Li}_{3} \mathrm{~N}$ was seen, and unique to PTMC:LiTFSI was $\mathrm{Li}_{2} \mathrm{~S}$. PEO was found to be more stable in regards to decomposition than PCL, and both of these systems were more stable than PTMC. Depending on the polymer system, the distribution between degradation products is leaning either towards polymer or salt degradation products, where polymer degradation is preferred for PCL:LiTFSI, while degradation of the TFSI anion dominates for the PEO and PTMC systems. In the latter system, the decomposition of both salt and polymer chains is more severe than in the other systems. The evolution of the Li 1s peak over time showed rapid formation of $\mathrm{Li}^{+}$compounds from the deposited metallic lithium, with gradual movement of the formed $\mathrm{Li}^{+}$compounds into the bulk of the materials. The kinetics of this process also showed 
large differences depending on the polymer system; with metallic lithium and only limited transport of $\mathrm{Li}^{+}$compounds into the bulk being detected for PTMC:LiTFSI, whereas the Li 1s signal was seen to return to the bulk position on a timescale of a few hours for both PEO:LiTFSI and PCL:LiTFSI. The combination of pronounced salt and polymer degradation with an apparent stability over time in contact with metallic lithium for PTMC:LiTFSI suggests the formation of an apparently robust interphase, indicating that designing an electrochemically robust polymer electrolyte system involves not only detecting the degradation, but also carefully characterizing the formed degradation layers. The results further show a clear depth dependence of different compounds, where salt accumulation is found prior to lithium depositions. Also, the decomposition products are generally found dominantly at the outermost surface region. However, as the amount of deposited lithium is very small (a few monolayers), it is not possible to determine if continuous breakdown would occur if the interface is contacted with a larger quantity of lithium. Further studies, where repeated lithium deposition is applied to the surface is needed for understanding the long-term stability of the decomposition layer in an operating battery.

\section{Experimental section}

\section{Materials}

$\varepsilon$-Caprolactone monomer (CL; Perstorp) was dried by distillation over $\mathrm{CaH}_{2}$ under reduced pressure. Lithium bis(trifluoromethanesulfonyl)imide salt (LiTFSI; BASF) was pre-dried at $120{ }^{\circ} \mathrm{C}$ under vacuum for $48 \mathrm{~h}$. Trimethylene carbonate (TMC; Richman Chemicals), tin(II)-2-ethylhexanoate catalyst ( SnOct $_{2}$; Sigma, 95\%), toluene (Acros Organics, 99.8\%, Super dry with molecular sieves), tetrahydrofuran (Sigma Aldrich, $>99.9 \%$, anhydrous, inhibitor-free), acetonitrile (Sigma Aldrich, 99.8\%, anhydrous), poly(ethylene oxide) (PEO; Sigma Aldrich, $M_{\mathrm{w}}$ : $2000000 \mathrm{~g} \mathrm{~mol}^{-1}$ ), were all used as-received. All chemicals were kept in inert argon atmosphere unless stated otherwise.

\section{Synthesis of PCL and PTMC}

High-molecular-weight poly(trimethylene carbonate) (PTMC) and poly( $\varepsilon$-caprolactone) (PCL) were prepared using the same bulk ring-opening polymerization described previously. ${ }^{26}$ In

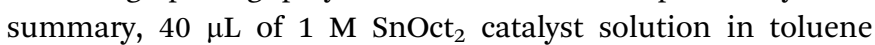
together with $200 \mathrm{mmol}$ of monomer were poured into a stainless steel reactor. The reactor was kept at $130{ }^{\circ} \mathrm{C}$ for $72 \mathrm{~h}$ for polymerization. The reactants were mixed by shaking the reactor every $30 \mathrm{~min}$ during the initial $90 \mathrm{~min}$. This method of synthesis yields a high molecular weight $\left(M_{\mathrm{w}} \sim 400000 \mathrm{~g} \mathrm{~mol}^{-1}\right.$ for PTMC and $\sim 700000 \mathrm{~g} \mathrm{~mol}^{-1}$ for PCL with a dispersity $\bigoplus_{\mathrm{M}} \sim$ 1.9 for both cases), and a low amount of residual monomer $(<10 \%){ }^{26,27}$ The glass transition temperature $\left(T_{\mathrm{g}}\right)$ of these are well below room temperature. ${ }^{28}$

\section{Polymer electrolyte preparation}

PEO, PCL or PTMC was dissolved in anhydrous solvent together with $25 \mathrm{wt} \%$ LiTFSI salt and stirred at $50{ }^{\circ} \mathrm{C}$ for $72 \mathrm{~h}$. The ratio of
PEO and PCL to tetrahydrofuran was 0.02 and $0.06 \mathrm{~g} \mathrm{~mL}^{-1}$, respectively. The ratio of PTMC to acetonitrile was $0.06 \mathrm{~g} \mathrm{~mL}^{-1}$. Next, approximately $50 \mu \mathrm{L}$ of polymer electrolyte solution was pipetted onto a stainless steel sample plate (Scienta Omicron). The solvent was slowly evaporated using a vacuum and heating procedure described previously. ${ }^{29}$ In summary, the pressure was slowly lowered to $<2$ mbar over $20 \mathrm{~h}$ at $30{ }^{\circ} \mathrm{C}$. Next, the temperature was increased to $60{ }^{\circ} \mathrm{C}$ and held for an additional $40 \mathrm{~h}$ at vacuum. Following evaporation of solvent, the samples were hot-pressed at $100{ }^{\circ} \mathrm{C}$ for $1 \mathrm{~h}$ and then left to cool in order to decrease the thickness further.

\section{Photoelectron spectroscopy}

Measurements were carried out at the CoESCA station ${ }^{30}$ on the UE52-PGM beamline ${ }^{31}$ at the BESSY II electron storage ring operated by the Helmholtz-Zentrum Berlin für Materialien und Energie. The UE52-PGM is a soft X-ray beamline installed at an APPLE-II-type undulator providing variable horizontal and elliptical polarized light in the energy range of 120-1600 eV. An angle-resolved time-of-flight spectrometer (ArTOF-II EW, Scienta Omicron) with a $4 \%$ energy window was used to measure the electron kinetic energy, all spectra shown are angleintegrated. The sample was placed in normal emission with respect to the analyzer. The experiment was performed during single-bunch operation using horizontal linear polarization. The base pressure during the experiment was in the mid $10^{-10}$ mbar range, all measurements were performed at room temperature. The photon bandwidth varied from $8 \mathrm{meV}$ at $250 \mathrm{eV}$ photon energy to $70 \mathrm{meV}$ at $1000 \mathrm{eV}$ photon energy, the spectrometer resolution at $300 \mathrm{eV}$ kinetic energy was approximately $0.2 \mathrm{eV}$ resulting in a total experimental broadening of $0.2 \mathrm{eV}$. To avoid exposure to the ambient atmosphere, the samples were transferred into the load lock in an argon atmosphere using a portable glovebag. To avoid radiation damage, the sample position was changed between measurements.

The core-level spectra for decomposition analysis were recorded with a $300 \mathrm{eV}$ kinetic energy resulting in similar escape depth for all samples. Energy calibration was done by measuring the $\mathrm{C}$ 1s spectra for the corresponding photon energies (see info below). These C 1s PES spectra were subsequently used for depth characterization. The photon energies were $(h v)$ of $1000 \mathrm{eV}, 845 \mathrm{eV}, 710 \mathrm{eV}, 600 \mathrm{eV}$ and $370 \mathrm{eV}$, the corresponding kinetic energies yields the inelastic mean free paths (IMFP, $\lambda$ ) of $2.3 \mathrm{~nm}, 1.9 \mathrm{~nm}, 1.5 \mathrm{~nm}, 1.2 \mathrm{~nm}$ and $0.4 \mathrm{~nm}$ (assuming primarily polymer in the near-surface region). ${ }^{32}$ This value corresponds to the IMFP in PCL but should cohere with that of PEO and PTMC adequately. A universal rule in PES is that $95 \%$ of information comes from the depth of $3 \lambda$ which would result in probing depths of $6.9 \mathrm{~nm}, 5.7 \mathrm{~nm}, 4.5 \mathrm{~nm}$, $3.6 \mathrm{~nm}$ and $1.2 \mathrm{~nm}$.

\section{In situ lithium deposition}

Lithium was deposited in situ onto the polymer film from a resistively heated Li-getter (SAES Getters) in the preparation chamber. Prior to the first deposition, the getter was degassed to ensure uncontaminated lithium deposition. The lithium 
deposition time was $5 \mathrm{~min}$ for all samples (current was set to 7.4 A), the pressure during deposition being approximately $1 \times$ $10^{-8}$ mbar.

\section{Data treatment and curve-fitting}

All data treatment was performed using Igor pro 6.37. The spectra were intensity normalized with respect to number of iterations, step size, dwell time and sweep acceptance. Binding energy calibration was done by aligning the LiTFSI peak to $293 \mathrm{eV}$ (ref. 14) in a reference C 1s spectrum acquired for every used photon energy. Where possible, the relative binding energy of known peaks, e.g. the multiple $\mathrm{C}$ 1s peak in the polymers, was locked in reference to a characteristic peak of the samples, to aid the fitting of the spectra.

\section{Conflicts of interest}

There are no conflicts to declare.

\section{Acknowledgements}

The Swedish Foundation for Strategic Research (project SOLID ALIBI, grant no. 139501338), Batteries Sweden (BASE), STandUP for Energy and Swedish Research Council (grant no. 201805336) are acknowledged for financial support. We thank the Helmholtz-Zentrum Berlin für Materialien und Energie for the allocation of synchrotron radiation beamtime.

\section{References}

1 E. P. Roth and C. J. Orendorff, Electrochem. Soc. Interface, 2012, 21, 45-49.

2 K. Xu, Chem. Rev., 2014, 114, 11503-11618.

3 J. Kalhoff, G. G. Eshetu, D. Bresser and S. Passerini, ChemSusChem, 2015, 8, 2154-2175.

4 J. Mindemark, M. J. Lacey, T. Bowden and D. Brandell, Prog. Polym. Sci., 2018, 81, 114-143.

5 J. Nair, L. Imholt, G. Brunklaus and M. Winter, Electrochem. Soc. Interface, 2019, 28, 55-61.

6 R. Bouchet, S. Lascaud and M. Rosso, J. Electrochem. Soc., 2003, 150, A1385-A1389.

7 P. Verma, P. Maire and P. Novák, Electrochim. Acta, 2010, 55, 6332-6341.

8 K. Takada, Acta Mater., 2013, 61, 759-770.

9 S. Wang, H. Xu, W. Li, A. Dolocan and A. Manthiram, J. Am. Chem. Soc., 2018, 140, 250-257.

10 C. Sångeland, J. Mindemark, R. Younesi and D. Brandell, Solid State Ionics, 2019, 343, 115068-115081.

11 K. Edström, M. Herstedt and D. P. Abraham, J. Power Sources, 2006, 153, 380-384.
12 S. Malmgren, K. Ciosek, M. Hahlin, T. Gustafsson, M. Gorgoi, H. Rensmo and K. Edström, Electrochim. Acta, 2013, 97, 23-32.

13 I. Ismail, A. Noda, A. Nishimoto and M. Watanabe, Electrochim. Acta, 2001, 46, 1595-1603.

14 C. Xu, B. Sun, T. Gustafsson, K. Edström, D. Brandell and M. Hahlin, J. Mater. Chem. A, 2014, 2, 7256-7264.

15 B. Sun, C. Xu, J. Mindemark, T. Gustafsson, K. Edström and D. Brandell, J. Mater. Chem. A, 2015, 3, 13994-14000.

16 C. Sångeland, B. Sun, D. Brandell, E. J. Berg and J. Mindemark, Batteries Supercaps, 2021, 4, 785-790.

17 M. Ebadi, C. Marchiori, J. Mindemark, D. Brandell and C. M. Araujo, J. Mater. Chem. A, 2019, 7, 8394-8404.

18 A. Mirsakiyeva, M. Ebadi, C. M. Araujo, D. Brandell, P. Broqvist and J. Kullgren, J. Phys. Chem. C, 2019, 123, 22851-22857.

19 S. Wenzel, T. Leichtweiss, D. Krüger, J. Sann and J. Janek, Solid State Ionics, 2015, 278, 98-105.

20 G. Beamson and D. Briggs, High Resolution XPS of Organic Polymers: The Scienta ESCA300 Database, Wiley, 1992.

21 P. C. Howlett, E. I. Izgorodina, M. Forsyth and D. R. MacFarlane, Z. Phys. Chem., 2006, 220, 1483-1498.

22 S. Randström, M. Montanino, G. B. Appetecchi, C. Lagergren, A. Moreno and S. Passerini, Electrochim. Acta, 2008, 53, 6397-6401.

23 C. F. N. Marchiori, R. P. Carvalho, M. Ebadi, D. Brandell and C. M. Araujo, Chem. Mater., 2020, 32, 7237-7246.

24 L. Suo, Y.-S. Hu, H. Li, M. Armand and L. Chen, Nat. Commun., 2013, 4, 1481.

25 J. Maibach, I. Källquist, M. Andersson, S. Urpelainen, K. Edström, H. Rensmo, H. Siegbahn and M. Hahlin, Nat. Commun., 2019, 10, 3080.

26 B. Sun, J. Mindemark, K. Edström and D. Brandell, Solid State Ionics, 2014, 262, 738-742.

27 C. Sångeland, T. Tjessem, J. Mindemark and D. Brandell, J. Phys. Chem. Lett., 2021, 12, 2809-2814.

28 J. Mindemark, B. Sun, E. Törmä and D. Brandell, J. Power Sources, 2015, 298, 166-170.

29 J. Mindemark, E. Törmä, B. Sun and D. Brandell, Polymer, 2015, 63, 91-98.

30 T. Leitner, A. Born, I. Bidermane, R. Ovsyannikov, F. O. L. Johansson, Y. Sassa, A. Föhlisch, A. Lindblad, F. O. Schumann, S. Svensson and N. Mårtensson, J. Electron Spectrosc. Relat. Phenom., 2021, 250, 147075.

31 D. R. Batchelor, T. Schmidt, R. Follath, C. Jung, R. Fink, M. Knupfer, A. Schöll, T. Noll, F. Siewert, B. Büchner and E. Umbach, Nucl. Instrum. Methods Phys. Res., Sect. A, 2007, 575, 470-475.

32 P. J. Cumpson, Surf. Interface Anal., 2001, 31, 23-34. 